\title{
Older Patients' Compliance with Drug Storage Recommendations
}

\author{
Nicolaas D. Vlieland ${ }^{1}$ - Bart J. F. van den Bemt ${ }^{2,3,4}$ - Charlotte L. Bekker ${ }^{1,2}$. \\ Marcel L. Bouvy ${ }^{5}$ Toine C. G. Egberts ${ }^{1,5} \cdot$ Helga Gardarsdottir ${ }^{1,5}$
}

Published online: 8 February 2018

(C) The Author(s) 2018. This article is an open access publication

\begin{abstract}
Background Whereas storage conditions are regulated and closely monitored in every stage of the drug supply chain before drug dispensing, it is unknown if patients store drugs at home according to storage recommendations.

Objective The objective of this study was to investigate how older patients store their prescription drugs at home and to what extent they comply with drug storage recommendations.

Methods We conducted a cross-sectional study between October 2015 and March 2016. Forty-four participating Dutch community pharmacies selected four home-dwelling patients (aged $\geq 65$ years) using at least one prescription drug. A complete drug inventory at patients' homes was
\end{abstract}

Electronic supplementary material The online version of this article (https://doi.org/10.1007/s40266-018-0524-8) contains supplementary material, which is available to authorized users.

Helga Gardarsdottir

h.gardarsdottir@umcutrecht.nl

Nicolaas D. Vlieland

N.D.Vlieland@umcutrecht.nl

1 Department of Clinical Pharmacy, Division Laboratory and Pharmacy, University Medical Center Utrecht,

Heidelberglaan 100, 3584 CX Utrecht, The Netherlands

2 Department of Pharmacy, Sint Maartenskliniek, Nijmegen, The Netherlands

3 Department of Pharmacy, Radboud Medical Center, Nijmegen, The Netherlands

4 Department of Clinical Pharmacy and Toxicology, Maastricht University Medical Center, Maastricht, The Netherlands

5 Division of Pharmacoepidemiology and Clinical Pharmacology, Utrecht Institute for Pharmaceutical Sciences, Utrecht University, Utrecht, The Netherlands performed. Drugs were considered to fulfill the storage recommendation when these met all drug quality $(\mathrm{Q})$ and information (I) criteria: adequately stored according to drug product label storage recommendations for temperature, light, humidity $(\mathrm{Q} 1)$; expiry date not passed $(\mathrm{Q} 2)$; integer primary package (Q3); drug identifiability (I1); drug package insert or information leaflet availability (I2). Results One hundred and seventy patients $[53.5 \%$ female, mean age 74.9 (standard deviation 7.3) years] were included and 1133 prescription drugs stored at home were registered. More than half of the patients $(51.2 \%)$ complied with all storage quality and information criteria. Assessment of the individual criteria showed that $76.4 \%$ of patients were compliant with criterion Q1 while 90.6, 95.3, 97.1 and $71.2 \%$ of patients complied with criteria Q2, Q3, I1 and I2, respectively. $53.2 \%$ of drugs that should be kept refrigerated according to storage criterion Q1 were not stored between 2 and $8{ }^{\circ} \mathrm{C}$.

Conclusion This study illustrates that more than half of the older patients comply with general drug storage recommendations.

\section{Key Points}

Compliance with storage recommendations was observed in more than half of the older patients.

Older patients storing more than five different drugs at home are more often non-compliant with storage recommendations.

Drugs requiring refrigeration were often not stored according to the recommended storage conditions. 


\section{Introduction}

Drug use increases with age, and it is estimated that $25-40 \%$ of patients aged above 65 years use at least five prescription drugs [1]. The use of multiple drugs increases the risk for several drug-related problems, such as nonadherence with drugs and storing expired drugs at home $[2,3]$. A recent study on home storage conditions of biological drugs showed that only $7 \%$ of patients stored these continuously at temperatures specified in the product label [4].

Proper drug storage conditions and practices at home are an important aspect of safe and effective drug treatment. The storage and distribution of drugs are strictly regulated and closely monitored in every stage of the drug supply chain as specified in the Good Distribution Practice guideline [5]. Patients are expected to store their drugs at home according to the storage conditions stated in the Summary of Product Characteristics, such as in the case of drugs requiring refrigeration or storage in the original (outer) packaging to protect from moisture or light, which are provided by the drug companies in the package insert and on the drugs' packaging. In addition to adequate storage conditions, patients should use the drug before the expiry date and keep the drug in an undamaged primary package to ensure drug quality. Furthermore, adequate storage practices also require patients to have access to drug information, by having drugs stored that are identifiable (e.g. for caretakers) and having package inserts available.

Older patients often use multiple drugs and are likely to have more difficulties with drug management at home, including storage, owing to visual or cognitive impairment [6-8]. Increased knowledge on home storage practices could help pharmacists to identify which drug products and aspects of home storage need more attention when counselling patients. This study aims to investigate how older patients store their drugs at home, and to what extent patients comply with drug product storage recommendations.

\section{Methods}

\subsection{Setting and Study Population}

This cross-sectional study was performed between October 2015 and March 2016. The participating pharmacists from 44 Dutch community pharmacies (recruited through the community pharmacist specialist education network) [9] each selected four home-dwelling patients aged 65 years or older who filled at least one prescription drug during the study period. These patients were invited either by telephone or face to face by the community pharmacist to participate. Eligible patients received both written and oral information about the study and were asked for written informed consent to participate.

\subsection{Ethics}

The Medical Ethics Review Committee of the University Medical Center Utrecht (protocol reference number 15-587/C) judged that the Medical Research Involving Human Subjects Act (WMO) was not applicable to this study. Anonymity of participants was ensured as no research data were traceable by the investigators.

\subsection{Study Procedure}

The community pharmacist visited each consenting patient twice at the patients' home. During the first visit, information on home storage of medication was collected using a structured drug inventory assessment (see Appendix S1 of the Electronic Supplementary Material). Patients were asked to present all prescription drugs and show all home storage locations of prescription drugs. The following characteristics were collected for each prescription drug at each storage location on the drug inventory form: drug name, marketing authorisation number (Dutch RVG 'Register Verpakte Geneesmiddelen' or European Union number), Anatomical Therapeutic Chemical (ATC) classification [10], amount (number of packages), use of the drug (chronic/as needed/ stopped), storage location, packaging condition (original; intactness), product insert or information leaflet present (yes/ no) and the expiry date (month/year). A small temperature logger [11] was placed at each drug storage location to measure storage temperatures. Pharmacists assessed every storage location for possible exposure to light or moisture. Patients received a questionnaire that included questions on socio- and demographic variables. These were collected a week later during the second visit along with the temperature loggers. Date and time of logger placement and collection were registered by the visiting pharmacist.

\subsection{Outcomes}

The assessment of patients' compliance with storage recommendations was performed for each drug based on patients' compliance with five criteria representing (Q) drug quality and (I) drug information availability: (Q1) appropriateness of the storage conditions; (Q2) drug had not passed expiry date; (Q3) primary package integrity; (I1) extent of identifiability of the drug; and (I2) availability of drug information. Patients were considered to comply with appropriate storage conditions (Q1) when storage temperatures for all drugs did not exceed the 
advised storage temperature range and the drugs were not stored in a humid place or exposed to light when applicable. Drugs not requiring refrigeration were considered to require storage at room temperature defined as temperature below $25{ }^{\circ} \mathrm{C}$ without excursions above $25{ }^{\circ} \mathrm{C}$ for $2 \mathrm{~h}$ or longer. Refrigerator storage was considered adequate if the temperature was between 2 and $8{ }^{\circ} \mathrm{C}$, without excursions outside this range for $2 \mathrm{~h}$ or longer.

The Dutch G Standard database (a database containing all drug products that are dispensed by or used in the pharmacy in the Netherlands) was used to extract and link the specific drug storage recommendations [12] for each drug. Storage temperatures were assessed for the following storage locations: kitchen, refrigerator, living room, bedroom, bathroom and other (e.g. basement, hallway). Drugs requiring refrigeration stored outside the refrigerator and in use (e.g. insulin pens) were considered adequately stored if this was allowed for the specific drug. With regard to special storage conditions for light and moisture, only drugs (independently of package status) that explicitly required no exposure to light or moisture were taken into account. Humidity and light exposure, at each storage location, were assessed by the pharmacist and defined as adequate or inadequate. Drugs were considered not expired (Q2) if the expiry dates of all drugs had not passed on the day of the first visit. The drugs' primary package integrity (Q3) was based on the intactness assessment of the primary package (e.g. damaged blister package).

Patients were considered to comply with the criteria of drug identifiability (I1) if drugs stored were identifiable by their primary or secondary packaging as assessed by the pharmacist. Drug storage in multi-dose dispensing systems up to several weeks was considered adequate. Drug information availability (I2) was considered adequate when the patient could present at least one insert for each drug. The primary outcome of this study was the proportion of patients who were compliant with all five criteria mentioned above for all prescription drugs they stored at home.

\subsection{Covariates}

Covariates included the patient characteristics sex, age (65-69, 70-74, $\geq 75$ years), family status (alone, with partner/others), educational level (low, medium, high), number of drugs stored at home $(<5, \geq 5)$, and storage locations $(1,2, \geq 3)$.
Fig. 1 Flowchart of patients, drug information forms and number of packages categorised for actual use

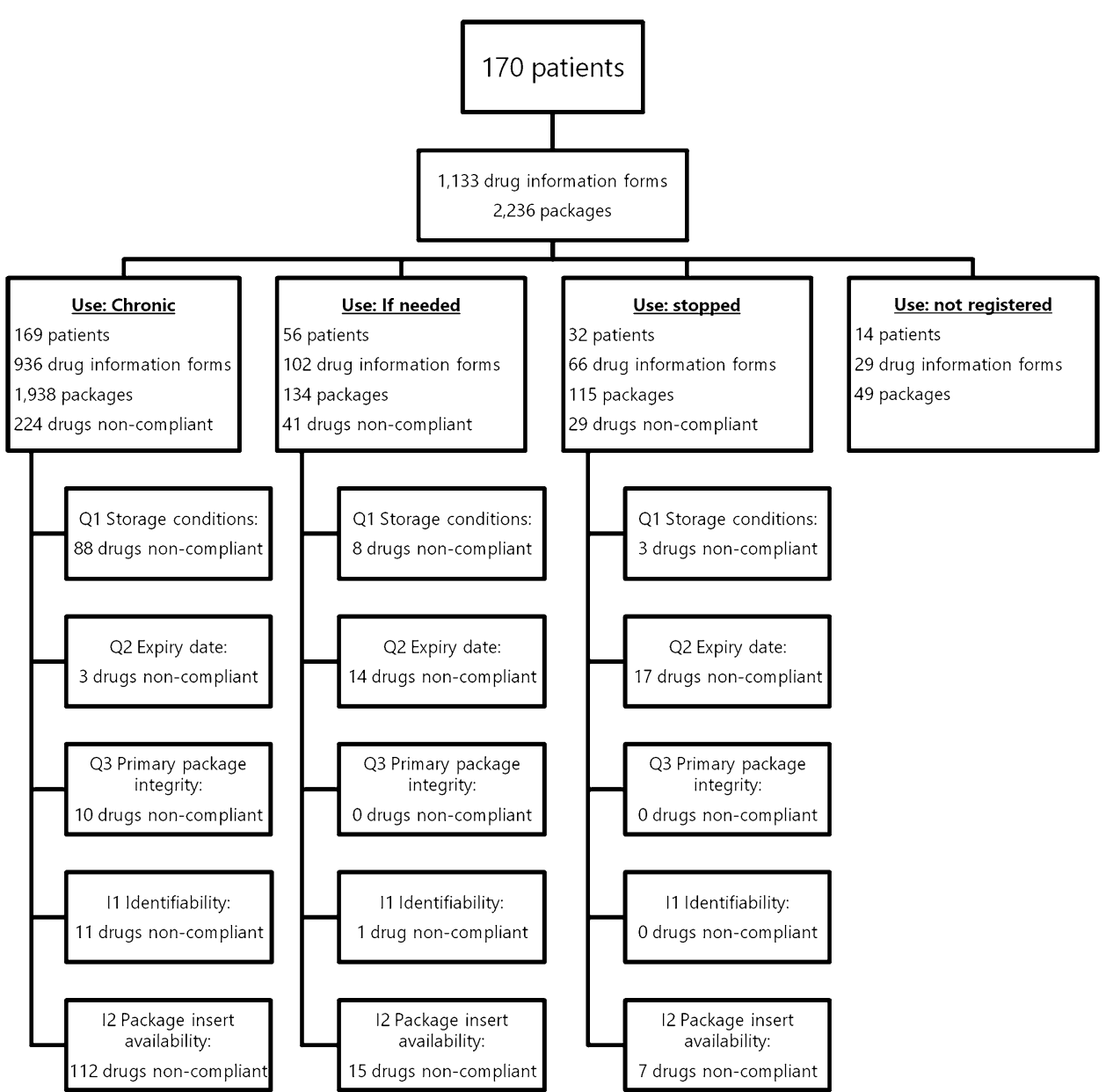




\subsection{Data Analysis}

Demographic data, temperature measurements and compliance with storage criteria were presented using means (standard deviation), medians (interquartile range) or in proportions of the study population. A multivariate logistic regression model was used to assess the associations between the primary outcome (patient compliance with storage conditions) and socio- and demographic variables, number of drugs stored at home, number of home storage locations (excluding 'refrigerator' location) and having drugs that require refrigeration. Results were presented as odds ratios (ORs) with 95\% confidence intervals (CIs) and adjusted for all covariates in the model. For the analysis of patient compliance with all storage criteria, only drugs that were in use ('chronic' and 'as needed') were assessed. All statistical analyses were performed using the statistical packages of SAS Version 9.4 (SAS Institute, Cary, NC, USA).

\section{Results}

One hundred and seventy patients were included in the study for which 1133 drug inventory forms were completed (Fig. 1). Slightly more than half of the patients were female $(53.5 \%), 47.1 \%$ aged 75 years or older and $34.1 \%$ of the patients were living alone. $16.5 \%$ of the patients had a low educational level. Drugs stored at patients' homes were most commonly agents acting on the renin-angiotensin system (55.0\%), drugs used for acid-related disorders (48.5\%), antithrombotic agents (47.9\%) and lipid-modifying agents (47.9\%) (Table 1). Patients stored most drugs in the kitchen (56.2\%), the bedroom (37.3\%) and the living room $(33.1 \%)$. Figure 2 illustrates examples of different storage locations used by patients in the study.

More than half (51.2\%) of the patients complied with all storage criteria for drugs they used long term or as needed. 69.4 and $70.0 \%$ of patients complied with drug quality criteria and drug information criteria, respectively. Figure 3 presents the level of compliance in proportions of
Table 1 Baseline characteristics of the study population $(n=170)$

\begin{tabular}{lc}
\hline Characteristic & Patients, $n(\%)$ \\
\hline Sex & \\
Female & $91(53.5)$ \\
Male & $79(46.5)$ \\
Age, years & \\
65-69 & $49(28.8)$ \\
$70-74$ & $41(24.1)$ \\
$\geq 75$ & $80(47.1)$ \\
Educational level & \\
Low & $28(16.5)$ \\
Medium & $94(55.3)$ \\
High & $33(19.4)$ \\
Family type & \\
Living alone & $58(34.1)$ \\
Living with partner/family member/other & $110(64.7)$ \\
Drug types (by ATC level 2) most frequently stored ${ }^{\mathrm{b}}$ & \\
C09 agents acting on the renin-angiotensin system & $93(55.0)$ \\
A02 drugs for acid-related disorders & $82(48.5)$ \\
C10 lipid-modifying agents & $81(47.9)$ \\
B01 Antithrombotic agents & $81(47.9)$ \\
C03 Diuretics & $73(43.2)$ \\
C07 Beta-blocking agents & $68(40.2)$ \\
C08 Calcium channel blockers & $42(24.9)$ \\
R03 drugs for obstructive airway diseases & $35(20.7)$ \\
C01 cardiac therapy & $27(16.0)$ \\
A10 drugs used in diabetes & $26(15.3)$ \\
\hline ATC Anatomical Thep
\end{tabular}

ATC Anatomical Therapeutic Chemical classification

${ }^{a}$ Numbers do not add up to $100 \%$ because of missing values

${ }^{\mathrm{b}}$ Top 10 , in descending order 

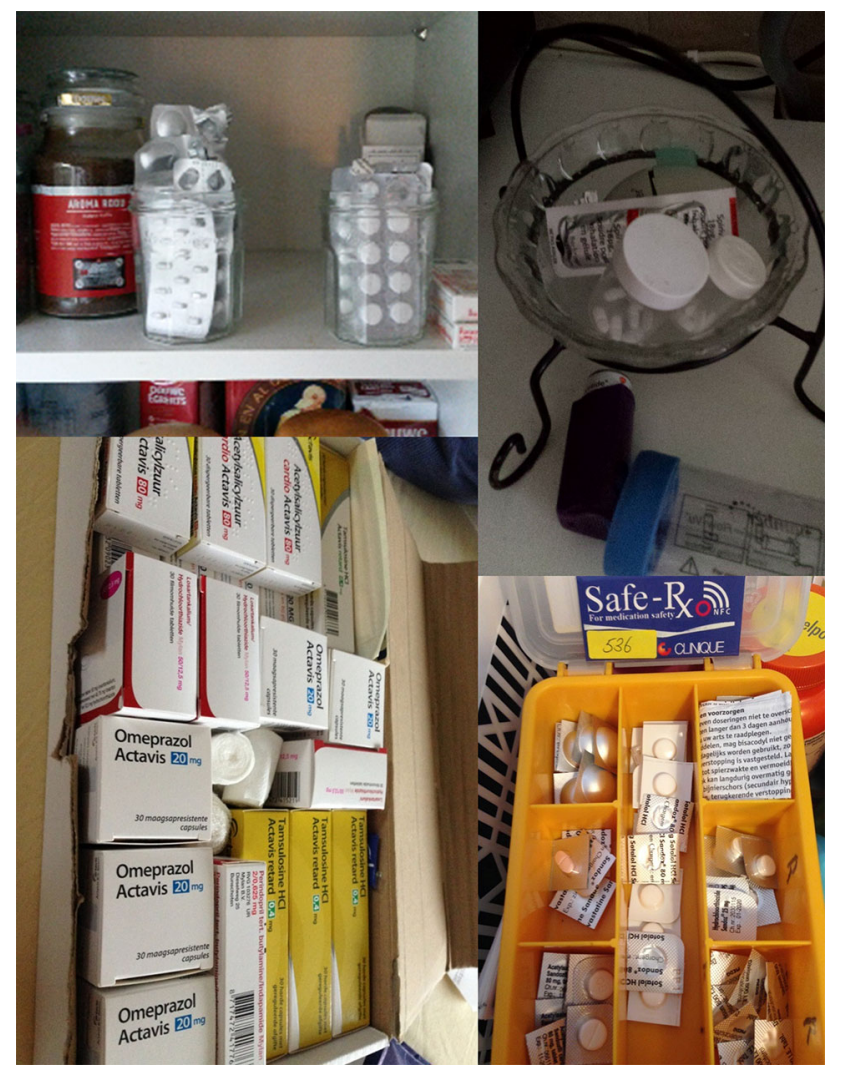

Fig. 2 Examples of drug storage at patients' homes

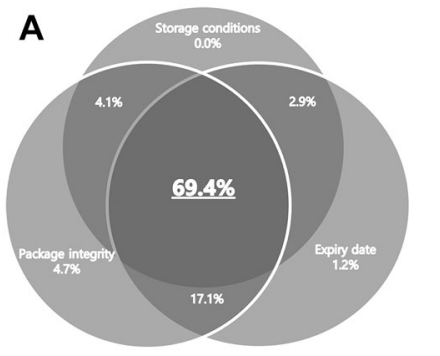

Q: drug quality criteria (\% pts compliant) Storage conditions (Q1) $\quad-76.4 \%$ Package integrity (Q3) $\quad-95.3 \%$

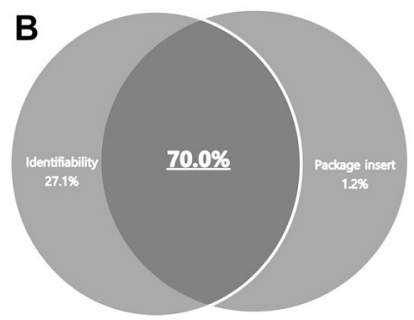

I: drug information criteria (\% pts compliant) Identifiability (11) Package insert (12) $-97.1 \%$ $-71.2 \%$

Fig. 3 Percentages of patients (pts) compliant with a the Q: drug quality criteria and $\mathbf{b}$ the I: drug information criteria (percentages add up to $100 \%$; e.g. $17.1 \%$ of patients comply with criteria Q2 and Q3, but not with criterion Q1). Total percentages of pts compliant with each criterion are shown on the right side. Three pts (1.8\%) did not comply with both drug information criteria (I1-2) and one patient $(0.6 \%)$ did not comply with the drug quality criteria (Q1-3) patients, one patient $(0.6 \%)$ did not comply with the drug quality criteria (Q1-3) (Fig. 3a) and three patients (1.8\%) did not comply with both drug information criteria (I1-2) (Fig. 3b).

One hundred and thirty (76.4\%) patients stored all drugs according to the recommended storage conditions for temperature, humidity and light exposure (Q1). Twentythree $(13.6 \%)$ patients stored drugs in a humid or light environment when the package label advised otherwise. Only one of these patients did not store a drug in the protective primary or secondary packaging (omeprazole outside primary and secondary packaging stored in a humid bathroom). There were 17 (9.4\%) patients who were using drugs that had already passed the expiry date (Q2). This mostly (82.4\%) concerned drugs that were used on an 'as needed' basis. One hundred and sixty-two (95.3\%) patients complied with primary package integrity (Q3). Drug information criteria are presented in Fig. 3b; 165 (97.1\%) patients stored all drugs in identifiable packaging (I1) and $121(71.2 \%)$ patients had the product insert or drug information leaflet (I2) available for all drugs at home.

The mean storage temperature of drugs was highest for drugs stored in the living room (mean $20.4^{\circ} \mathrm{C}$, range 13.0-27. ${ }^{\circ} \mathrm{C}$ ) and lowest in the bedroom (mean $17.7^{\circ} \mathrm{C}$, range 8.4-28.6 ${ }^{\circ} \mathrm{C}$ ) (Table 2). Drugs requiring refrigeration were stored at a mean temperature of $9.1^{\circ} \mathrm{C}$ (range -0.3 to $14.5^{\circ} \mathrm{C}$ ) and $53.2 \%$ of these drugs did not comply with the drug quality criteria. Drugs most often not stored according to storage recommendations were calcium/vitamin D preparations, omeprazole and levothyroxine, with 35.5, 34.1 and $33.3 \%$ of these drugs, respectively, not stored in compliance with at least one of the five storage criteria.

As presented in Table 3, the number of drugs stored at a patient's home was associated with non-compliance with one or more storage criteria (five or more drugs $\mathrm{OR}=2.21,95 \%$ CI 1.08-4.50). Having at least one drug that required refrigeration was associated with non-compliance with one or more storage criteria (adjusted $\mathrm{OR}=3.63 ; 95 \%$ CI 1.12-11.74). Furthermore, patients storing drugs at three or more locations at home showed an almost three-fold increase (crude $\mathrm{OR}=2.62,95 \% \mathrm{CI}$ 1.01-6.82) in non-compliance with one or more storage criteria, but this did not reach significance in the adjusted model (adjusted OR $=2.34,95 \%$ CI 0.82-6.70). Sex, age, family type and educational level were not associated with patients' compliance with one or more storage criteria.

\section{Discussion}

This study illustrates that more than half of the older patients store their drugs according to general storage recommendations. $53.2 \%$ of drugs requiring refrigeration 
Table 2 Prescription drugs stored at home including storage locations, compliance with storage criteria and storage temperature

\begin{tabular}{llccc}
\hline Storage location & Patients, $n(\%)$ & Number of drugs ${ }^{\mathrm{a}}, n(\%)$ & $\begin{array}{l}\text { Number of drugs non-compliant } \\
\text { with storage criteria }{ }^{\mathrm{b}}, n(\%)\end{array}$ & $\begin{array}{l}\text { Storage temperature } \\
\left({ }^{\circ} \mathrm{C}\right), \text { mean }(\text { range })\end{array}$ \\
\hline Kitchen (excluding the refrigerator) & $95(56.2)$ & $383(40.9)$ & $95(24.8)$ & $20.2(7.6-30.3)$ \\
Refrigerator & $22(13.0)$ & $30(3.2)$ & $16(53.2)$ & $9.1(-0.3$ to -14.5$)$ \\
Bedroom & $63(37.3)$ & $230(24.6)$ & $38(16.4)$ & $17.7(8.4-28.6)$ \\
Living room & $56(33.1)$ & $253(27.0)$ & $35(14.0)$ & $20.4(13.0-27.1)$ \\
Bathroom & $33(19.5)$ & $75(8.0)$ & $21(28.0)$ & $19.1(10.1-24.4)$ \\
Other (e.g. hallway, basement) & $38(22.5)$ & $188(20.1)$ & $47(25.2)$ & $19.7(8.3-33.9)$ \\
\hline
\end{tabular}

${ }^{a}$ Percentages add up to $>100 \%$ because of multiple storage locations for the same type of drug

${ }^{\mathrm{b}}$ Percentages of the number of drugs non-compliant/total number of drugs

were not stored according to the recommended storage conditions. Patients with at least five prescription drugs or having at least one drug that requires refrigeration often do not comply with storage recommendations.

The majority of patients in our study population had one or more drugs intended for treating chronic diseases stored at home, such as drugs intended for the treatment of cardiovascular diseases or respiratory diseases. These findings are in accordance with reports on the most commonly dispensed drugs in the Netherlands [1]. Several studies from different countries have investigated home storage of drugs, including Mexico, Malaysia, United Arab Emirates, Belgium and Greece. All identified several problems related to the home storage of drugs [13-17], such as overdue expiry dates and other undesirable storage practices (e.g. storing large quantities of drugs and maintaining drugs that were no longer used). The number of patients storing expired drugs in our study is slightly lower than what others report (5-20\%) [16, 18, 19]. This was also observed for the availability of package inserts [16] and might be partially explained by the fact that we included only prescription drugs and the fact that patients stored fewer drugs at home that were used on an 'as needed' basis or that were already discontinued. Having multiple drugs stored at multiple locations has been associated with poor medication storage practices [3, 19]. For older patients, it is thought that their cognitive skills and knowledge to independently manage their drugs are decreased [7]. However, this was not confirmed in our study in which older patients (aged $\geq 75$ years) were found, although not statistically significantly so, to be more compliant with the storage criteria.

Based on the temperature measurements performed, most drugs intended for storage in the refrigerator were not stored between 2 and $8{ }^{\circ} \mathrm{C}$. This is in line with what has been reported previously regarding storage of drugs that require refrigeration [4]. Pharmacists and pharmacy assistants should explicitly inform patients about adequate cool storage conditions (e.g. central shelf in the refrigerator, away from the back wall) when a drug requiring refrigeration is dispensed at the pharmacy. If drug storage between 2 and $8{ }^{\circ} \mathrm{C}$ is necessary and there is evidence that drug products degrade rapidly, refrigerators should be equipped with temperature registration or the use of dedicated refrigerators for drug storage should be encouraged.

Few patients $(9.4 \%)$ stored drugs at home that had passed the expiry date. As one would expect, drugs used 'as needed' or that had been discontinued more often had passed the expiry date than drugs used long term. Good storage practices require patients to check expiry dates of drugs regularly, preventing the use of expired drugs. Although we did not study patients' habits in relation to drug package expiry dates, others have reported that less than half of patients never check the drugs' expiry date before use [7,20]. More than $28 \%$ of patients did not have the package insert and drug information leaflets available for at least one drug. Many patients stored drug information leaflets and package inserts at a central location. Although the absence of the package insert may not directly affect treatment, accurate information about drugs that patients take long term or may intend to take as needed should be readily available. Primary package integrity is essential for some drugs to protect against light or moisture. Drug identifiability (e.g. storage in recognisable packaging) allows patients and caregivers to identify the drugs and the dosage regimen. In our study, however, only a small number of patients had one or more drugs in a damaged primary packaging $(4.7 \%)$ or had drugs at home that were not identifiable by the pharmacist (2.9\%).

The clinical consequences of not complying with storage recommendations are largely unknown. In theory, drugs exposed to inadequate storage conditions can lose 
Table 3 Associations between patient characteristics and compliance with drug storage criteria

\begin{tabular}{|c|c|c|c|c|c|c|c|}
\hline & $\begin{array}{l}\text { Patients } \\
(170), \\
n(\%)\end{array}$ & $\begin{array}{l}\text { Patients }(87) \\
\text { compliant for all } \\
\text { drugs, } n(\%)\end{array}$ & $\begin{array}{l}\text { Patients }(83) \text { with } \geq 1 \\
\operatorname{drug}(\mathrm{s}) \text { non-compliant, } \\
n(\%)\end{array}$ & $\begin{array}{l}\text { Drugs, } \\
n(\%)\end{array}$ & $\begin{array}{l}\text { Drugs non-compliant } \\
\text { with storage criteria, } \\
n(\%)\end{array}$ & $\begin{array}{l}\text { Crude odds } \\
\text { ratio }(95 \% \\
\text { CI) }\end{array}$ & $\begin{array}{l}\text { Adjusted odds } \\
\text { ratio }(95 \% \mathrm{CI})\end{array}$ \\
\hline \multicolumn{8}{|l|}{ Sex } \\
\hline Male & $79(46.5)$ & $45(51.7)$ & $34(41.0)$ & $439(44.7)$ & $110(25.1)$ & $\begin{array}{l}0.65 \\
\quad(0.35-1.19)\end{array}$ & $\begin{array}{l}0.65 \\
\quad(0.31-1.36)\end{array}$ \\
\hline \multicolumn{8}{|l|}{ Age, years } \\
\hline $65-69$ & $49(28.8)$ & $22(25.3)$ & $27(32.5)$ & $242(24.6)$ & $92(38.0)$ & Reference & Reference \\
\hline $70-74$ & $41(24.1)$ & $21(24.1)$ & $20(24.1)$ & $251(25.5)$ & $66(26.3)$ & $\begin{array}{l}0.78 \\
\quad(0.34-1.78)\end{array}$ & $\begin{array}{l}0.44 \\
\quad(0.22-1.46)\end{array}$ \\
\hline$\geq 75$ & $80(47.1)$ & $44(50.6)$ & $36(43.4)$ & $490(49.9)$ & $104(21.2)$ & $\begin{array}{l}0.67 \\
\quad(0.33-1.36)\end{array}$ & $\begin{array}{l}0.43 \\
\quad(0.21-1.11)\end{array}$ \\
\hline \multicolumn{8}{|c|}{ Family type $^{\mathrm{a}}$} \\
\hline Alone & $58(34.1)$ & $31(35.6)$ & $27(32.5)$ & $360(36.6)$ & $76(21.1)$ & $\begin{array}{l}0.87 \\
\quad(0.46-1.65)\end{array}$ & $\begin{array}{l}0.68 \\
\quad(0.31-1.48)\end{array}$ \\
\hline \multicolumn{8}{|c|}{ Educational level $^{\mathrm{a}}$} \\
\hline Low & $37(21.8)$ & $14(16.1)$ & $23(27.7)$ & $268(27.2)$ & $69(25.7)$ & Reference & Reference \\
\hline Medium & $94(55.3)$ & $48(55.2)$ & $46(55.4)$ & $511(52.0)$ & $141(27.6)$ & $\begin{array}{l}0.62 \\
\quad(0.26-1.47)\end{array}$ & $\begin{array}{l}0.73 \\
\quad(0.27-1.94)\end{array}$ \\
\hline High & $33(19.4)$ & $20(23.0)$ & 13 (15.7) & $165(16.8)$ & $51(30.9)$ & $\begin{array}{l}0.42 \\
\quad(0.15-1.18)\end{array}$ & $\begin{array}{l}0.41 \\
\quad(0.13-1.26)\end{array}$ \\
\hline \multicolumn{8}{|c|}{ Number of storage locations ${ }^{\mathrm{b}}$} \\
\hline 1 & $76(44.7)$ & $42(48.3)$ & $34(41.0)$ & $425(43.2)$ & $97(22.8)$ & Reference & Reference \\
\hline 2 & $69(40.6)$ & $37(42.5)$ & $32(38.6)$ & $379(38.6)$ & $98(25.9)$ & $\begin{array}{l}1.07 \\
\quad(0.56-2.06)\end{array}$ & $\begin{array}{l}1.24 \\
\quad(0.60-2.59)\end{array}$ \\
\hline $3-5$ & $25(14.7)$ & $8(9.2)$ & $17(20.5)$ & $179(18.2)$ & $67(37.4)$ & $\begin{array}{l}2.62 \\
\quad(1.01-6.82)\end{array}$ & $\begin{array}{l}2.34 \\
(0.82-6.70)\end{array}$ \\
\hline \multicolumn{8}{|c|}{ Number of drugs } \\
\hline$\geq 5$ & $98(57.6)$ & $41(47.1)$ & $57(68.7)$ & $791(80.5)$ & $215(27.2)$ & $\begin{array}{l}2.46 \\
(1.32-4.60)\end{array}$ & $\begin{array}{l}2.21 \\
\quad(1.08-4.50)\end{array}$ \\
\hline \multicolumn{8}{|c|}{ Drugs stored in the refrigerator } \\
\hline Yes & $20(11.8)$ & $5(5.7)$ & $15(18.1)$ & $179(18.2)$ & $69(38.5)$ & $\begin{array}{l}3.25 \\
\quad(1.12-9.40)\end{array}$ & $\begin{array}{l}3.63 \\
\quad(1.12-11.74)\end{array}$ \\
\hline
\end{tabular}

CI confidence interval

${ }^{a}$ Numbers do not add up to $100 \%$ because of missing values (family type: 2 values missing, educational level: 6 values missing)

${ }^{\mathrm{b}}$ Refrigerators were not taken into account as storage locations

efficacy or become toxic. There are few case reports and studies performed on suspected clinical consequences of inadequate drug storage. A physician reported a suspected drug therapy failure owing to inadequate storage of levothyroxine at the patient's home [21]. Several drugs such as tetracycline antibiotics are susceptible to temperature changes and moisture, but there are few cases known where outdated tetracycline antibiotics had clinical consequences likely caused by a degraded product [22]. Furthermore, low humidity storage conditions have been shown to compromise inhalation capsules [23]. In general, drug companies should use protective packaging to protect drugs if they are sensitive to moisture or light. It is therefore improbable that drugs in our inventory that were exposed to light or moisture and stored in the primary or secondary packaging (as most drugs in the inventory) were affected by these conditions.

Only a few studies have investigated different aspects of adequate storage conditions and included home visits by pharmacists to make a complete inventory of prescription drugs. This study provides more insight into storage practices at home and took into account a combination of important storage requirements, such as temperature measurements of storage locations, expiry dates, product insert availability and drug identifiability. However, this study was also subject to some limitations. Participants were aware of the study purpose and might have already discarded unused drugs and reorganised their household before the visit. This might have led to an underestimation of both the number of patients who store drugs 
inappropriately and the number of drugs not stored appropriately. In addition, there might have been differences in the type and level of questioning used by pharmacists in their attempts to make the inventory of all drugs in the home as complete as possible. Light exposure or relative humidity were also not measured but based on the assessment of the pharmacists. However, to limit variation in the amount of information per patient, pharmacists received protocol training and were required to use a standardised inventory list for each drug they found.

\section{Conclusion}

Compliance with storage recommendations was observed in more than half of the older patients. Patients taking multiple drugs and drugs requiring refrigeration are often non-compliant with storage recommendations. Drug companies and pharmacists should emphasise the importance of proper drug storage at home. All patients using multiple drugs may require additional help from their pharmacist, pharmacy technician or physician to store drugs at home appropriately. Extra and repeated information, both written and oral, on drug storage conditions (e.g. middle compartment of the refrigerator or do not store in the bathroom) should be provided to patients as deemed necessary for specific products.

Acknowledgements The authors thank all contributing pharmacies.

\section{Compliance with Ethical Standards}

Funding No sources of funding were used to assist in the conduct of this study or the preparation of this article.

Conflict of interest Nicolaas Vlieland, Bart van den Bemt, Charlotte Bekker, Antoine Egberts, Marcel Bouvy and Helga Gardarsdottir have no conflicts of interest directly relevant to the content of this study.

Open Access This article is distributed under the terms of the Creative Commons Attribution-NonCommercial 4.0 International License (http://creativecommons.org/licenses/by-nc/4.0/), which permits any noncommercial use, distribution, and reproduction in any medium, provided you give appropriate credit to the original author(s) and the source, provide a link to the Creative Commons license, and indicate if changes were made.

\section{References}

1. SFK. Data en feiten 2015. Het jaar 2014 in cijfers. 2015. http:// www.sfk.nl/nieuws-publicaties/data-en-feiten/data-en-feiten2015. Accessed 9 Feb 2017.

2. Wieczorkiewicz SM, Kassamali Z, Danziger LH. Behind closed doors: medication storage and disposal in the home. Ann Pharmacother. 2013;47(4):482-9.
3. Sorensen L, Stokes JA, Purdie DM, Woodward M, Roberts MS. Medication management at home: medication risk factor prevalence and inter-relationships. J Clin Pharm Ther. 2006;31(5):485-91.

4. Vlieland ND, Gardarsdottir H, Bouvy ML, Egberts TC, van den Bemt BJ. The majority of patients do not store their biologic disease-modifying antirheumatic drugs within the recommended temperature range. Rheumatology (Oxf). 2016;55(4):704-9.

5. European Commission. Guidelines on good distribution practice of medicinal products for human use. 2013. http://ec.europa.eu/ health//sites/health/files/files/eudralex/vol-1/2013_c343_01/ 2013_c343_01_en.pdf. Accessed 9 Feb 2017.

6. Maddigan SL, Farris KB, Keating N, Wiens CA, Johnson JA. Predictors of older adults' capacity for medication management in a self-medication program: a retrospective chart review. J Aging Health. 2003;15(2):332-52.

7. Sino CG, Sietzema M, Egberts TC, Schuurmans MJ. Medication management capacity in relation to cognition and self-management skills in older people on polypharmacy. J Nutr Health Aging. 2014;18(1):44-9.

8. Notenboom K, Beers E, van Riet-Nales DA, Egberts TC, Leufkens HG, Jansen PA, et al. Practical problems with medication use that older people experience: a qualitative study. J Am Geriatr Soc. 2014;62(12):2339-44.

9. Koster ES, Blom L, Philbert D, Rump W, Bouvy ML. The Utrecht Pharmacy Practice Network for Education and Research: a network of community and hospital pharmacies in the Netherlands. Int J Clin Pharm. 2014;36(4):669-74.

10. World Health Organization. ATC/DDD index 2017. 2017. http:// www.whocc.no/atc_ddd_index/. Accessed 18 Apr 2017.

11. Confrerie Clinique. SafeRx. 2014. http://www.confrerie-clinique. com/products/tempos/. Accessed 3 Jan 2017.

12. Z-Index. G-Standaard. 2015. http://www.z-index.nl/g-standaard. Accessed 17 Jan 2017.

13. Gracia-Vasquez SL, Ramirez-Lara E, Camacho-Mora IA, CantuCardenas LG, Gracia-Vasquez YA, Esquivel-Ferrino PC, et al. An analysis of unused and expired medications in Mexican households. Int J Clin Pharm. 2015;37(1):121-6.

14. Ali SE, Ibrahim MI, Palaian S. Medication storage and selfmedication behaviour amongst female students in Malaysia. Pharm Pract (Granada). 2010;8(4):226-32.

15. Sharif SI, Abduelkarem AR, Bustami HA, Haddad LI, Khalil DS. Trends of home drug storage and use in different regions across the northern United Arab Emirates. Med Princ Pract. 2010;19(5):355-8.

16. De Bolle L, Mehuys E, Adriaens E, Remon JP, Van Bortel L, Christiaens T. Home medication cabinets and self-medication: a source of potential health threats? Ann Pharmacother. 2008;42(4):572-9.

17. Tsiligianni IG, Delgatty C, Alegakis A, Lionis C. A household survey on the extent of home medication storage: a cross-sectional study from rural Crete. Greece. Eur J Gen Pract. 2012;18(1):3-8.

18. Ewen S, Baumgarten T, Rettig-Ewen V, Mahfoud F, GrieseMammen N, Schulz M, et al. Analyses of drugs stored at home by elderly patients with chronic heart failure. Clin Res Cardiol. 2015;104(4):320-7.

19. Sorensen L, Stokes JA, Purdie DM, Woodward M, Roberts MS. Medication management at home: medication-related risk factors associated with poor health outcomes. Age Ageing. 2005;34(6):626-32.

20. Si PK, Koo KN, Doon D, Chew L. Knowledge of prescription medications among cancer patients aged 65 years and above. J Geriatr Oncol. 2012; 3(2):120-30.

21. Benvenga S. Solution of a diagnostic problem upon visiting the patient at home and reading their medication leaflet. Endocr Pract. 2014;20(5):517. 
22. Frimpter GW, Timpanelli AE, Eisenmenger WJ, Stein HS, Ehrlich LI. Reversible "Fanconi syndrome" caused by degraded tetracycline. JAMA. 1963;184:111-3.

23. Renswouw DC, Laarhoven AC, Haren MJ, Bouvy ML, Weda M. Storage instructions for inhalation capsules: consequences of incorrect storage and adherence in daily practice. J Pharm Pract. 2010;23(6):548-52. 